UDC 512.5

P. Zakrzewski (Inst. Math., Univ. Warsaw, Poland)

\title{
ON THE COMPLEXITY OF THE IDEAL OF ABSOLUTE NULL SETS*
}

\section{ПРО КОМПЛЕКСНІСТЬ ІДЕАЛУ АБСОЛЮТНИХ НУЛЬ-МНОЖИН}

Answering a question of Banakh and Lyaskovska, we prove that for an arbitrary countable infinite amenable group $G$ the ideal of sets having $\mu$-measure zero for every Banach measure $\mu$ on $G$ is an $F_{\sigma \delta}$ subset of $\{0,1\}^{G}$.

У відповідь на питання, поставлене Банахом і Лясківською, доведено, що для будь-якої зліченної аменабельної групи $G$ ідеал множин, що мають нульову $\mu$-міру для будь-якої міри Банаха $\mu$ на $G, € F_{\sigma \delta}$-підмножиною $\{0,1\}^{G}$.

1. Introduction. This note is related to a paper by T. Banakh and N. Lyaskovska [1]. Given an amenable group $G$, Banakh and Lyaskovska considered the ideal $\mathcal{N}$ of absolute null subsets of $G$, i.e., sets having $\mu$-measure zero for every Banach measure $\mu$ on $G$ (a finitely-additive, probability, left-invariant measure $\mu: \mathcal{P}(G) \rightarrow[0,1]$ defined on the family of all subsets of $G$; see [3]). Since each ideal on a countable infinite group $G$ can be considered as a subspace of the Cantor set $\{0,1\}^{G}$ it makes sense to consider its descriptive properties. Banach and Lyaskovska asked ([1], Problem 4) whether the ideal of absolute null subsets of the group $\mathbb{Z}$ is co-analytic. In this note we prove (see Corollary 3.1) that for an arbitrary countable infinite amenable group $G$ the ideal $\mathcal{N}$ is in fact $F_{\sigma \delta}$. This follows from a characterisation of absolute null subsets of an arbitrary amenable group (see Proposition 2.1) based on the notion of the intersection number of Kelly [2].

2. A characterisation of absolute null sets. Following Kelly [2] we define the intersection number $I(\mathcal{B})$ of a family $\mathcal{B}$ of subsets of a set $X$ to be $\inf \{i(S) / n(S)\}$ where the infimum is taken over all finite sequences $S=\left(S_{1}, \ldots, S_{n}\right)$ of (not necessary distinct) elements of $\mathcal{B}, n=n(S)$ is the length of $S$ and

$$
i(S)=\sup \left\{\sum_{i=1}^{n} \chi_{S_{i}}(x): x \in X\right\} .
$$

Proposition 2.1. Let $G$ be an amenable group and $A \subseteq G$. Then the following are equivalent:

(1) A is absolute null.

(2) The intersection number of the family $\{g A: g \in G\}$ is zero.

Proof. (1) $\Rightarrow$ (2). Assume that $I(\{g A: g \in G\})=\delta>0$. By a theorem of Kelly (see [2], Theorem 2), there is a finitely additive probability measure $m$ defined on $\mathcal{P}(G)$ such that $m(g A) \geq \delta$ for each $g \in G$.

Let $\theta$ be a Banach measure on $G$. Following the proof of Invariant Extension Theorem (see [4], Theorem 10.8) define a function $\mu: \mathcal{P}(G) \rightarrow[0,1]$ by letting

$$
\mu(B)=\int_{G} m\left(g^{-1} B\right) d \theta(g), \quad \text { for } \quad B \subseteq G .
$$

It is easy to see that $\mu$ is a Banach measure on $G$. Moreover, we have

$$
\mu(A)=\int_{G} m\left(g^{-1} A\right) d \theta(g) \geq \inf \left\{m\left(g^{-1} A\right): g \in G\right\} \geq \delta>0,
$$

which shows that $A \notin \mathcal{N}$.

\footnotetext{
${ }^{*}$ This research was partially supported by MNiSW Grant Nr N N201 543638.
} 
(2) $\Rightarrow(1)$. Let $\mu$ be an arbitrary Banach measure on $G$. Suppose that $\mu(A)=\epsilon>0$. Then, since $\mu$ is left-invariant, we also have $\mu(g A)=\epsilon$ for every $g \in G$. Consequently, by [2] (Proposition 1), $I(\{g A: g \in G\}) \geq \epsilon>0$.

Proposition 2.1 is proved.

3. The Borel complexity of the ideal $\mathcal{N}$. The following corollary of Proposition 2.1 gives an answer to a question of Banakh and Lyaskovska (see [1], Problem 4).

Corollary 3.1. Let $G$ be an amenable group and $A \subseteq G$. Then the following are equivalent:

(1) A is absolute null.

(2) $\forall k \in \mathbb{N} \exists n \in \mathbb{N} \exists \bar{g} \in G^{n+1} \forall S \subseteq\{1, \ldots, n+1\}$ :

$$
\frac{|S|}{n+1}>\frac{1}{k+1} \Rightarrow \bigcap_{i \in S} g_{i} A=\varnothing .
$$

In particular, if $G$ is countably infinity, then formula (2) gives a $F_{\sigma \delta}$ definition of the ideal $\mathcal{N}$.

Proof. It is easy to see that formula (2) simply states that $I(\{g A: g \in G\})=0$ so its equivalence with condition (1) was established in Proposition 2.1.

To prove the remaining part of the corollary, assume that $G$ is countably infinity. Then it is enough to show that for fixed $n \in \mathbb{N}, \bar{g} \in G^{n+1}$ and $S \subseteq\{1, \ldots, n+1\}$ the family $\left\{A \subseteq G: \bigcap_{i \in S} g_{i} A=\varnothing\right\}$ is closed in $\mathcal{P}(G)$.

But this follows from the fact that for $A \subseteq G$ we have

$$
\bigcap_{i \in S} g_{i} A=\varnothing \Longleftrightarrow \forall g \in G \exists i \in S: g_{i}^{-1} g \notin A .
$$

Corollary 3.1 is proved.

4. Some open problems. Let $G$ be an arbitrary infinite group. Following a suggestion by Taras Banakh (personal communication) let us call a set $A \subseteq G$ Kelly null if the intersection number of the family $\{g A: g \in G\}$ is zero; denote by $\mathcal{K}$ the collection of all Kelly null subsets of $G$. In view of Proposition 2.1, $\mathcal{K}$ is an ideal of subsets of $G$ provided the group $G$ is amenable. On the other hand, Proposition 5.1 of [1] implies that if $G$ has a free subgroup of rank 2, then $\mathcal{K}$ is not an ideal; in fact $G$ is then the union of two Kelly null sets. In any case, however, $\mathcal{K}$ contains a (possibly proper) subfamily $\mathcal{A}_{\mathcal{K}}=\{A \subseteq G: \forall K \in \mathcal{K} K \cup A \in \mathcal{K}\}$ which already forms an ideal.

The remarks above lead to the following problems suggested by Banakh.

Problem 1. Characterise groups $G$ for which $\mathcal{K}$ is an ideal.

Problem 2. Characterise groups $G$ which are finite unions of elements of $\mathcal{K}$.

Problem 3. Given a countably infinite group $G$ find a combinatorial description of elements of the ideal $\mathcal{A}_{\mathcal{K}}$. What is its descriptive complexity? In particular, is it Borel?

Acknowledgements. The author would like to thank Taras Banakh for his valuable comments and the suggestions above.

1. Banakh T., Lyaskovska N. Completeness of translation-invariant ideals on groups // Ukr. Mat. Zh. - 2010. - 62, № 8. - P. $1022-1031$.

2. Kelley J. L. Measures on Boolean algebras // Pacif. J. Math. - 1959. - 9. - P. 1165-1177.

3. Paterson A. Amenability. - Amer. Math. Soc., 1988.

4. Wagon S. The Banach - Tarski paradox // Encyclopedia Math. and Its Appl. - Cambridge Univ. Press, 1986. 